\title{
LMI-Based Robust Controllers for DC-DC Cascade Boost Converters
}

\author{
Carlos Andrés Torres-Pinzón*, Roberto Giral", and Ramon Leyva ${ }^{\dagger}$ \\ ${ }^{\dagger *}$ Departament d’Enginyeria Electrònica, Elèctrica, i Automàtica, Escola Tècnica Superior d’Enginyeria, Universitat \\ Rovira i Virgili, Tarragona, Spain
}

\begin{abstract}
This paper presents two different robust controllers for boost converters with two stages in a cascade. The first robust controller is monovariable; that is, the duty-cycle is the same for the two switches. The monovariable controller ensures that some prescribed constraints on pole placement and control effort are met, and optimizes the load disturbance rejection, while takes into account the uncertainty in certain parameters. The first controller is then compared with a multivariable robust controller; that is, with independent duty cycles in each switch. The multivariable controller takes into account the same uncertainty, constraints and optimization function. The comparison shows that the multivariable controller performs better at the expense of a slightly more complex implementation; that is, the multivariable controller provides a better rejection of the load disturbance. The paper also describes simulations and experimental results that are in perfect agreement with theoretical derivations.
\end{abstract}

Key words: Boost-boost converters, DC-DC converters, Cascade converters, LMI, Quadratic converters, Robust control, Uncertainty

\section{INTRODUCTION}

Basic DC-DC converters have practical limitations for voltage conversion when the ratio between the output voltage and the input voltage is too high or too low [1]. This is the reason why we use other topologies when a wide conversion ratio is required. An alternative for wide voltage conversion are converters with transformer like the reported in [14]. Nevertheless, using transformers could involve dangerous voltage spikes that limit the efficiency and causes undesirable noise [15], [16]. When non-isolation is required an efficient alternative to the basic topologies are cascade topologies. An example of cascade topology is the cascade buck converter where the conversion ratio is the product of the duty-cycle of each stage. The cascade buck converter is a good choice to wide-range voltage reductions, as for instance in present microprocessors, which need to be fed with a very low voltage between $3.3 \mathrm{~V}$ and $1.5 \mathrm{~V}$ [2]. Another example of cascade topologies is the cascade boost converter that is used when a

Manuscript received Aug. 3, 2011; revised Jun. 1, 2012

Recommended for publication by Associate Editor Byung-Cho Choi.

${ }^{\dagger}$ Corresponding Author: ramon.leyva@urv.cat

Tel: +34 977558520, Fax: +34 977559605, Universitat Rovira i Virgili

* Departament d'Enginyeria Electrònica, Elèctrica, i Automàtica, Escola

Tècnica Superior d’Enginyeria, Universitat Rovira i Virgili, Spain large voltage step-up is required, for example, in certain renewable energy applications where the voltage of the renewable source is very low and the required output voltage is high [3], [13].

The cascade converters have multiple stages that are driven by their respective duty cycles. Therefore, cascade converters are inherently multivariable systems. However, in order to simplify the controller implementation, most of the authors report controllers for these systems where duty-cycles are equal for all of the stages [1], [2], [8]; thus the system becomes monovariable.

Control based on linear matrix inequalities (LMI) provides a solution that ensures to comply with a great number of design requirements where classical techniques fail to obtain an analytical solution [4]. LMI control technique does not provide an analytical solution but a numerical one that is achieved using modern numerical optimization methods to ensure the control constraints and thus to find the controller gains.

This fact has prompted some authors to apply LMI control in the dc-dc converter field [6], [7], [17], [18]. This technique allows us to ensure the satisfaction of requirements on stability, closed-loop pole placement and control effort and, simultaneously, to maximize the level of disturbance rejection. In [6], [7], Olalla et alt. analyze LMI controllers for boost 


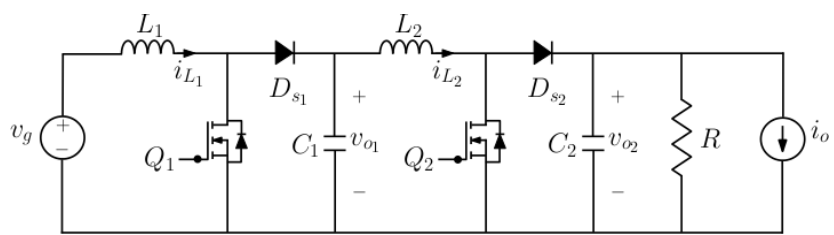

Fig. 1. Schematic circuit of a cascade boost converter.

converters optimizing the load disturbance rejection and describe their implementation. In [17], Montagner analyzes LMI controllers where the parameters depend on measurable converter parameters. In all the previous contributions the converter has a single stage, thus the control is monovariable. Unlike the previous works, we present an LMI controller which is multivariable for cascade converters and compare it with a monovariable alternative. We verify that the multivariable alternative performs better, whereas the design procedure involves a similar optimization program and the controller implementation is only slightly more complex.

The paper is organized as follows, Section II reviews the modeling of the boost converter with two stages in a cascade when the duty cycles of the two switches are equal and when they are independent. The modeling takes into account the uncertainty in converter parameters. Section III describes the LMI design requirements used in the control design; that is, the maximum level of disturbance rejection, the pole placement restriction and the bounding in control effort. In Section IV, the control procedure is explained and some simulations are provided. The experimental verifications are shown in section V. Finally, section VI summarizes the main conclusions.

\section{DC-DC CASCADE BoOst CONVERTER MODEL}

This section presents the averaged dynamic model of the cascade boost converter and describes the uncertainty of the converter parameters by means of a polytopic representation.

\section{A. Averaged Model of Cascade Boost Converters}

Fig. 1 shows the schematic of a boost converter with two stages in cascade, where $V_{g}$ is the input voltage, $i_{0}$ is the load current disturbance, and $v_{01}$ and $v_{02}$ are the capacitor voltages. $R$ models the converter nominal load, while $L_{1}, L_{2}, C_{1}$, and $C_{2}$ stand for the inductances and capacitances values, respectively. The state variables are the inductor currents $i_{L 1}$ and $i_{L 2}$ and the capacitor voltages $v_{01}$ and $v_{02}$; thus the state vector corresponds to $x(t)=\left[\begin{array}{llll}i_{L 1}(t) & v_{o 1}(t) & i_{L 2}(t) & v_{o 2}(t)\end{array}\right]$.

The binary signals $u_{1}$ and $u_{2}$ turn the MOSFETs ON and OFF at a constant switching frequency $1 / T_{s}$. $T_{o n_{1}}$ and $T_{\text {off }}$ are the time intervals at which the switch Q1 remains ON $\left(u_{1}=1\right)$ and $\operatorname{OFF}\left(u_{1}=0\right)$, respectively. We use the same convention for
$T_{\mathrm{on}_{2}}\left(u_{2}=1\right)$ and $T_{\text {off } 2}\left(u_{2}=0\right)$ of switch Q2. The switching period holds that $T_{s}=T_{o n_{1}}+T_{o f f_{1}}=T_{o n 2}+T_{o f f_{2}}$ and we have synchronized the beginning of both ON intervals $T_{o n_{1}}$ and $T_{o n_{2}}$. The ratios $d_{1}=T_{o n_{1}} / T_{s}$ and $d_{2}=T_{o n_{2}} / T_{s}$ are the duty cycles of the first and second switch, respectively. We assume that the converter operates in continuous conduction mode.

The dynamic behavior of the cascade boost converter at each position of the switches can be obtained by using the Kirchhoff's laws. Thus, the dynamic expressions in state-space at each position of the switch set is characterized as,

$$
\begin{array}{lll}
\dot{x}(t)=A_{1} x(t)+B_{1} & \text { during } & \left(T_{\text {off } 1} T_{\text {on } 2}\right) \\
\dot{x}(t)=A_{2} x(t)+B_{2} & \text { during } & \left(T_{\text {on } 1} T_{\text {on } 2}\right) \\
\dot{x}(t)=A_{3} x(t)+B_{3} & \text { during } & \left(T_{\text {on } 1} T_{\text {off } 2}\right) \\
\dot{x}(t)=A_{4} x(t)+B_{4} & \text { during } & \left(T_{\text {off } 1, \text { off } 2}\right)
\end{array}
$$

where $A_{\mathrm{i}}$ and $B_{\mathrm{i}}(i \in\{1,2,3,4\})$ are the state matrices and the input vector, respectively, for each subinterval. Also, $x$ is the state vector which groups the inductor currents and capacitor voltages $x=\left[\begin{array}{llll}i_{L 1} & v_{o 1} & i_{L 2} & v_{02}\end{array}\right]^{T}$.

Thus, expression (1) can be compacted in the following manner,

$$
\begin{aligned}
\dot{x}(t)= & \left(A_{1} x(t)+B_{1}\right)\left(1-u_{1}\right) u_{2}+\left(A_{2} x(t)+B_{2}\right) u_{1} u_{2}+ \\
& \left(A_{3} x(t)+B_{3}\right) u_{1}\left(1-u_{2}\right)+\left(A_{4} x(t)+B_{4}\right)\left(1-u_{1}\right)\left(1-u_{2}\right)
\end{aligned}
$$

Therefore, the converter dynamics can be written as

$$
\dot{x}(t)=\left[\begin{array}{cccc}
0 & -\frac{1-u_{1}}{L_{1}} & 0 & 0 \\
\frac{1-u_{1}}{C_{1}} & 0 & -\frac{1}{C_{1}} & 0 \\
0 & \frac{1}{L_{2}} & 0 & -\frac{1-u_{2}}{L_{2}} \\
0 & 0 & -\frac{1-u_{2}}{C_{2}} & -\frac{1}{R C_{2}}
\end{array}\right] x(t)+\left[\begin{array}{c}
\frac{V_{g}}{L_{1}} \\
0 \\
0 \\
-\frac{i_{o}}{C_{2}}
\end{array}\right]
$$

This switched model can be approximated by using the state-space averaging model [5], [9], which is obtained by replacing the binary signals $u_{1}$ and $u_{2}$ by their duty cycles $d_{1}$ and $d_{2}$, and the state vector by their corresponding averaged values during the switching period.

1) Monovariable linearized averaged model: We consider a single duty cycle, that is $d=d_{1}=d_{2}$, and a single output signal $v_{o 2}(t)$ that corresponds with the output capacitor voltage, in the monovariable model. We also consider an additional state variable $x_{5}$ to take into account the integral of the output voltage error $x_{5}=\int_{0}^{t}\left(v_{o 2}(\tau)-V_{\text {ref }}\right) d \tau$, to ensure a zero output error in steady state. Consequently, 
$v_{o 2}=V_{\text {ref }}=V_{g} / D^{\prime 2}$ in equilibrium, where $D^{\prime}=1-D$ is the switches complementary steady-state duty cycle. We also consider a disturbance signal corresponding to an additional output current $i_{o}$. Therefore, the augmented averaged model of the monovariable cascade boost converter can be written as

$$
\begin{aligned}
& \dot{\tilde{x}}(t)=A^{\text {mono }} \tilde{x}(t)+B_{w}^{\text {mono }} \tilde{w}(t)+B_{d}^{\text {mono }} \tilde{d}(t) \\
& \tilde{z}(t)=C_{z}^{\text {mono }} \tilde{x}(t)+D_{z w}^{\text {mono }} \tilde{w}(t)+D_{z d}^{\text {mono }} \tilde{d}(t)
\end{aligned}
$$

where $\tilde{x}, \tilde{d}$, and $\tilde{w}$ are the averaged values of the state vector, duty cycle and disturbance in incremental form; that is, $\tilde{x}=\langle x-X\rangle_{T_{\mathrm{s}}}, \quad \tilde{d}=\langle d-D\rangle_{T_{\mathrm{s}}}, \quad \tilde{w}=i_{o}$, being

$$
\begin{aligned}
& A^{\text {mono }}=\left[\begin{array}{ccccc}
0 & -\frac{D^{\prime}}{L_{1}} & 0 & 0 & 0 \\
\frac{D^{\prime}}{C_{1}} & 0 & -\frac{1}{C_{1}} & 0 & 0 \\
0 & \frac{1}{L 2} & 0 & \frac{D^{\prime}}{L_{2}} & 0 \\
0 & 0 & \frac{D^{\prime}}{C_{2}} & -\frac{1}{R C_{2}} & 0 \\
0 & 0 & 0 & 1 & 0
\end{array}\right] \quad B_{d}^{\text {mono }}=\left[\begin{array}{c}
\frac{V_{\text {ref }} D^{\prime}}{L_{1}} \\
-\frac{V_{\text {ref }}}{R C_{1} D^{\prime 2}} \\
\frac{V_{\text {ref }}}{L_{1}} \\
-\frac{V_{\text {ref }}}{R C_{2} D^{\prime}} \\
0
\end{array}\right] \quad B_{w w}^{\text {mono }}=\left[\begin{array}{c}
0 \\
0 \\
0 \\
-\frac{1}{C_{2}} \\
0
\end{array}\right] \\
& C_{z}^{\text {mono }}=\left[\begin{array}{lllll}
0 & 0 & 0 & 1 & 0
\end{array}\right] \quad D_{z d}^{\text {mono }}=[0] \quad D_{z w}^{\text {mono }}=[0]
\end{aligned}
$$

and the equilibrium state vector expression

$$
X=\left[\begin{array}{lllll}
\frac{V_{g}}{R D^{\prime 4}} & \frac{V_{g}}{D^{\prime}} & \frac{V_{g}}{R D^{\prime 2}} & \frac{V_{g}}{D^{\prime 2}} & 0
\end{array}\right]^{T}
$$

In the following subsections, we consider as uncertain values the load $R$ and the steady-state complementary duty cycle $D^{\prime}$, hence matrices $A^{\text {mono }}$ and $B_{u}^{\text {mono }}$ are uncertain.

2) Multivariable linearized averaged model: In the multivariable model, we consider a multiple input; that is, the input vector corresponds to $\tilde{d}=\left[\begin{array}{ll}\tilde{d}_{1} & \tilde{d}_{2}\end{array}\right]^{T}$ and we take as output vector the voltages in capacitors $\tilde{z}=\left[\begin{array}{ll}\tilde{V}_{o 1} & \tilde{V}_{o 2}\end{array}\right]^{T}$, where $\tilde{v}_{o 1}=v_{o 1}-\frac{V_{\text {ref }}}{2}$ and $\tilde{v}_{o 2}=v_{o 2}-V_{\text {ref }}$. In order to ensure zero steady-state output errors we add two new additional variables $x_{5}=\int_{0}^{t}\left(v_{o 2}(\tau)-V_{\text {ref }}\right) d \tau$ and $x_{6}=\int_{0}^{t}\left(v_{o 2}(\tau)-V_{\text {ref }}\right) d \tau$. Thus, the augmented averaged model of the multivariable cascade boost converter can be derived from (3) as,

$$
\begin{aligned}
& \dot{\tilde{x}}(t)=A^{\text {multi }} \tilde{x}(t)+B_{w}^{\text {multi }} \tilde{w}(t)+B_{d}^{\text {multi }} \tilde{d}(t) \\
& \tilde{z}(t)=C_{z}^{\text {mult }} \tilde{x}(t)+D_{z w}^{\text {multi }} \tilde{w}(t)+D_{z d}^{\text {mult }} \tilde{d}(t)
\end{aligned}
$$

being

$$
A^{\text {muliti }}=\left[\begin{array}{cccccc}
0 & -\frac{D_{1}^{\prime}}{L_{1}} & 0 & 0 & 0 & 0 \\
\frac{D_{1}^{\prime}}{C_{1}} & 0 & -\frac{1}{C_{1}} & 0 & 0 & 0 \\
0 & \frac{1}{L 2} & 0 & \frac{D_{2}^{\prime}}{L_{2}} & 0 & 0 \\
0 & 0 & \frac{D_{2}^{\prime}}{C_{2}} & -\frac{1}{R C_{2}} & 0 & 0 \\
0 & 1 & 0 & 0 & 0 & 0 \\
0 & 0 & 0 & 1 & 0 & 0
\end{array}\right]
$$

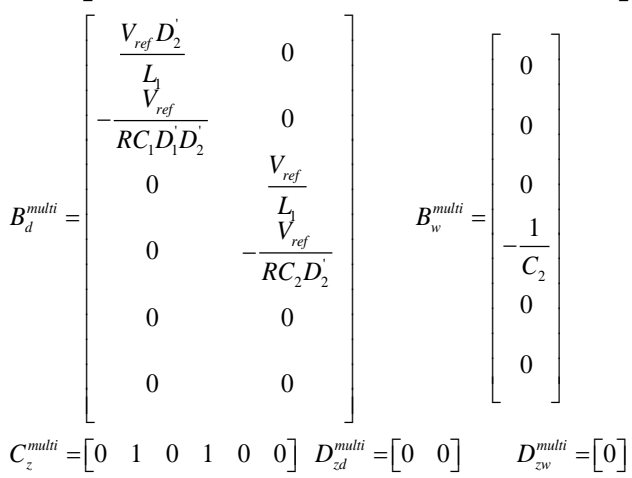

and the multivariable equilibrium state vector

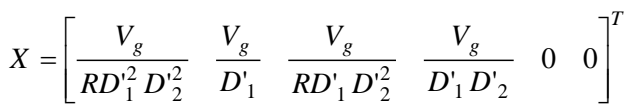

In the multivariable model, we consider as uncertain values the load $R$ and the steady-state complementary duty-cycle $D_{1}^{\prime}$, hence matrices $A^{\text {multi }}$ and $B_{u}^{\text {multi }}$ are uncertain. It is worth to note that since $v_{o 1}=\frac{V_{\text {ref }}}{2}$ and $v_{o 2}=V_{\text {ref }}$ in steady state, $D_{2}^{\prime}=0.5$ does not depend on the value of the input voltage $V_{g}$, therefore there is no uncertainty in parameter $D_{2}^{\prime}$.

\section{B. Uncertain Polytopic Representation of the Cascade Boost Converter}

Since some converter parameters are uncertain, some terms of dynamic expressions (4) and (7) are only known inside a certain interval. Thus, in order to ensure that the control requirements are met for any value of the parameters, we define a vector $\rho$ which groups the uncertain terms such that the matrices $A$ and $B$ have a linear dependence on these $\rho$ parameters. Then we define a convex polytope that contains all the possible values of dynamic matrices $A(\rho)$ and $B(\rho)$.

1) Uncertain polytope in monovariable model: Since the uncertain converter parameters are $R$ and $D^{\prime}$ in this model, a $\rho$ vector such as that groups the terms $\left\{\frac{1}{R}, D^{\prime}, \frac{1}{R D^{\prime}}, \frac{1}{R D^{\prime 2}}\right\}$ thus ensuring a linear dependence of 
$A(\rho)$ and $B_{d}(\rho)$ on $\rho$.

Therefore, the possible values of $\rho$ are hold within a polytope of $L=2^{4}$ vertices $\left\{v_{1}, \ldots, v_{16}\right\}$ such that its coordinates are bounded by

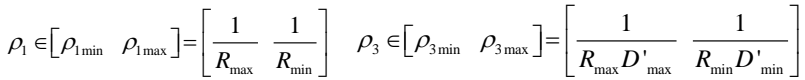

$\rho_{2} \in\left[\begin{array}{ll}\rho_{2 \min } & \rho_{2 \max }\end{array}\right]=\left[\begin{array}{ll}D_{\min }^{\prime} & D_{\max }^{\prime}\end{array}\right] \rho_{4} \in\left[\begin{array}{ll}\rho_{4 \min } & \rho_{4 \max }\end{array}\right]=\left[\begin{array}{ll}\frac{1}{R_{\max } D_{\max }^{\prime 2}} & \frac{1}{R_{\min } D_{\text {min }}^{\prime 2}}\end{array}\right](10)$

The convex polytope that contains all the possible values of $\rho$ can be expressed as the following convex combination [4]

$$
\rho \in C_{o}\left\{v_{1}, \ldots, v_{16}\right\}=\left\{\sum_{i=1}^{16} \lambda_{i} v_{i}, \quad \lambda_{i} \geq 0, \sum_{i=1}^{16} \lambda_{i}=1,\right\}
$$

And, because of the linear dependence on $\rho$, the system matrices $A(\rho)$ and $B_{d}(\rho)$ are contained in the following convex polytope

$\left[A(\rho), B_{d}(\rho)\right] \in C_{o}\left\{\varsigma_{1}, \ldots, \varsigma_{16}\right\}=\left\{\sum_{i=1}^{16} \lambda_{i} \varsigma_{i}, \lambda_{i} \geq 0, \sum_{i=1}^{16} \lambda_{i}=1\right\}$

where the vertex $\varsigma_{i}$ corresponds to the image of $v_{i}$, that is $\varsigma_{i}=\left[A\left(v_{i}\right), B\left(v_{i}\right)\right]$. Thus using the concept differential inclusion [4], we ensure the fulfillment of control requirements for the whole family of system matrices $\left[A(\rho), B_{d}(\rho)\right]$.

It is worth to note that, despite this model has two uncertain parameters, the vector $\rho$ has four dimensions so that the system matrices is linearly dependent on $\rho$. This procedure involves some degree of conservatism but it will ensure the robustness of the control.

2) Uncertain polytope in multivariable model: The uncertain converter parameters in the multivariable model are $R$ and $D^{\prime}{ }_{1}$, and the chosen $\rho$ vector for a linear dependence groups the terms $\left\{\frac{1}{R}, D_{1}^{\prime}, \frac{1}{R D_{1}^{\prime}}\right\}$.

Consequently, the possible values of $\rho$ are hold within a polytope of $2^{3}$ vertices $\left\{v_{1}, \ldots, v_{8}\right\}$ such that its coordinates are inside the intervals

$$
\begin{aligned}
& \rho_{1} \in\left[\begin{array}{ll}
\rho_{1 \text { min }} & \rho_{1 \max }
\end{array}\right]=\left[\begin{array}{ll}
\frac{1}{R_{\max }} & \frac{1}{R_{\min }}
\end{array}\right] \\
& \rho_{2} \in\left[\begin{array}{ll}
\rho_{2 \min } & \rho_{2 \max }
\end{array}\right]=\left[\begin{array}{ll}
D_{1 \min }^{\prime} & D_{1 \max }^{\prime}
\end{array}\right] \\
& \rho_{3} \in\left[\begin{array}{ll}
\rho_{3 \min } & \rho_{3 \max }
\end{array}\right]=\left[\begin{array}{ll}
\frac{1}{R_{\max } D_{1 \max }^{\prime}} & \frac{1}{R_{\min } D_{1 \min }^{\prime}}
\end{array}\right]
\end{aligned}
$$

Therefore the system matrices $A(\rho)$ and $B(\rho)$ of the multivariable model are contained inside the corresponding convex polytope.

In next section, we apply the control requirements to the previous uncertain models.

\section{LMI DESIGN CONSTRAINTS}

\section{A. Introduction}

In this subsection, we revisit some concepts of LMI control that are next applied to cascade boost converters.

LMI has being used in control for a long time, a classical control theorem establishes that the system

$$
\dot{\tilde{x}}(t)=A \tilde{x}(t)
$$

is stable i.e., all the trajectories converge to zero, quadratically stable in Lyapunov sense, if and only if there exists a positive-definite matrix $P$ (i.e., $P>0$ ), such that

$$
A^{T} P+P A<0
$$

Therefore the stability requirement has become a problem of solving a linear matrix inequality whose variable is $P$. Here $>$ means positive definite and $<$ means negative definite. It is worth to note that we have removed the superindex mono or multi since we can consider any of the previous models.

This concept can be extended to a family of systems defined by a polytopic representation, thus ensuring the robust stability despite of the particular value of the system matrices. That is, let the family of systems

$$
\dot{\tilde{x}}(t)=A(\rho) \tilde{x}(t)
$$

where $\rho$ is an unknown parameter of $A$, assuming that $A(\rho)$ are within a convex polytope of matrices with vertices $A_{i}$, that is, $A(\rho) \in C_{o}\left\{A_{1}, \ldots, A_{L}\right\}$ or equivalently, $A(\rho)$ can be expressed as a convex combination of $A$

$$
A(\rho)=\sum_{i=1}^{L} \lambda_{i} A_{i}, \quad \lambda_{i} \geq 0, \sum_{i=1}^{L} \lambda_{i}=1
$$

Then, we can prove the stability of the system family (16) by regarding if there exists $P>0$ such that

$$
A^{T}(\rho) P+P A(\rho)<0
$$

The previous expression can be rewritten as

$$
\left(\sum_{i=1}^{L} \lambda_{i} A_{i}^{T}\right) P+P\left(\sum_{i=1}^{L} \lambda_{i} A_{i}\right)<0
$$

or equivalently,

$$
\sum_{i=1}^{L} \lambda_{i}\left(A_{i}^{T} P+P A_{i}\right)<0
$$

Therefore, if there exists a symmetric matrix $P>0$ that meets quadratic stability in each vertex

$$
A_{i}^{T} P+P A_{i}, \quad i=1, \ldots, L .
$$


the system is stable with independence of the unknown value of $\rho$.

This concept can also be extended to feedback controlled systems, in the following manner. Given the system

$$
\dot{\tilde{x}}(t)=A \tilde{x}(t)+B_{d} \tilde{d}(t)
$$

where $\tilde{d}(t)$ is the input signal and we assume a linear feedback law $\tilde{d}(t)=F \tilde{x}(t)$, then the closed-loop system is stable if there exists a $P>0$ such that

$$
\left(A+B_{d} F\right)^{T} P+P\left(A+B_{d} F\right)<0
$$

Nevertheless, when $P$ and $F$ are variables of expression (23), the inequality is nonlinear. However, it can be rewritten as

$$
A W+W A^{T}+B_{d} Y+Y^{T} B_{d}<0
$$

where $W=P^{-1}, Y$ is defined so that $F=Y W^{-1}$, and thus, expression (24) is an LMI. Hence, we can obtain all the feedback gain vectors $F$ that stabilize the system (22) by finding all $W$ and $Y$ that fulfill (24).

The stability constraint can be extended to a family of systems $\left[A(\rho), B_{d}(\rho)\right]$ imposing the restriction (24) to each vertex $\left[A_{i}, B_{d i}\right]$.

Other restrictions can be imposed on the feedback gain vector $F$ to ensure, in addition to stability, an appropriate dynamic behavior in closed-loop. These restrictions are studied in the following subsection.

\section{B. LMI Constraints on Design Requirements}

We analyze the $H \infty$ performance, the pole placement and the control effort as requirements that should be imposed in the controller design.

1) LMI Formulation for $H$ Control Design $H^{\infty}$ : We consider that the feedback controlled system (24) with a linear feedback $\tilde{d}(t)=F \tilde{x}(t)$ is affected by a disturbance signal $\tilde{w}(t)$, that is

$$
\begin{aligned}
& \dot{\tilde{x}}(t)=\left(A+F B_{d}\right) \tilde{x}(t)+B_{w} \tilde{w}(t) \\
& \tilde{z}(t)=\left(C_{z}+D_{z d} B_{d}\right) \tilde{x}(t)+D_{z w} \tilde{w}(t)
\end{aligned}
$$

We desire to impose restrictions on the controller gain vector $F$ such that the energy gain of the output $\tilde{z}(t)$ is not larger than a certain value $\gamma$, that is

$$
\|\tilde{z}\|_{2}<\gamma\|\tilde{w}\|_{2} \quad \forall \tilde{w} \in L_{2}
$$

This is equivalent to the next restriction in the frequency domain [4],

$$
\max _{\omega}|G(j \omega)|<\gamma
$$

being $G(j \omega)$ the transfer function $G(j \omega)=C(j \omega I-A)^{-1} B_{d}+D_{z d}$.

This means that any frequency of the disturbance signal will not be amplified more that $\gamma$.

Conditions (26) and (27) correspond with the $H_{\infty}$ norm of the transfer function $G$. The restriction is met if there exists a Lyapunov function $V(x)=x^{T} P x$ that comply with the following inequality [4]

$$
\frac{d}{d t} V(x)+z^{T} z-\gamma^{2} w^{T} w \leq 0
$$

with $P>0$ and $\gamma>0$.

The preceding inequality can be expressed in an equivalent manner by means of the following LMI

$$
\left[\begin{array}{ccc}
\mathrm{AW}+\mathrm{WA}^{\mathrm{T}}+\mathrm{B}_{\mathrm{u}} \mathrm{Y}+\mathrm{Y}^{\mathrm{T}} \mathrm{B}_{u}^{\mathrm{T}} & B_{w} & \mathrm{WC}_{\mathrm{z}}^{\mathrm{T}}+\mathrm{Y}^{T} \mathrm{D}_{z \mathrm{u}}^{\mathrm{T}} \\
B_{w}^{T} & -\gamma I & 0 \\
\mathrm{C}_{\mathrm{z}} \mathrm{W}+\mathrm{D}_{\mathrm{zu}} Y & 0 & -\gamma I
\end{array}\right]<0
$$

where $W=P^{-1}$ and $Y=F W$.

This result is readily extended to uncertain systems with a polytopic representation [7], [11].

2) LMI Formulation for pole-placement: Another important constraint to be imposed to a family of systems dynamics is the pole placement. We desire that the closed-loop poles are inside a prescribed [11], [7]. This region ensures a minimum decay rate $\alpha$, a minimum damping ratio $\zeta=\sin (\theta)$ and a maximum natural frequency $\omega_{0}=r$. Thus, this region bounds the maximum overshoot, the rising time and the settling time.

The constraint of decay rate is imposed by means of the following LMI,

$$
A W+W A^{T}+B_{u} Y+Y B_{u}+2 \alpha W<0
$$

The damping ratio is limited by the LMI

$$
\left[\begin{array}{cc}
\cos (\theta)\left(A W+W A^{T}+B_{u} Y+Y^{T} B_{u}^{T}\right) & \sin (\theta)\left(A W-W A^{T}+B_{u} Y-Y^{T} B_{u}^{T}\right) \\
\sin (\theta)\left(-A W+W A^{T}-B_{u} Y+Y^{T} B_{u}^{T}\right) & \cos (\theta)\left(A W+W A^{T}+B_{u} Y+Y^{T} B_{u}^{T}\right)
\end{array}\right]<0
$$

In addition, bounds on the natural frequency involve another LMI

$$
\left[\begin{array}{cc}
-r W & W A^{T}+Y^{T} B_{u}^{T} \\
A W+B_{u} Y & -r W
\end{array}\right]<0
$$

A detailed explanation about the previous LMIs and their extension to a family of systems can be found in [11].

3) LMI Formulation for constraint on control input: A control fulfilling with all the previous restrictions but presenting an excessive gain $F$ would be affected by the duty-cycle saturation, which would worsen the expected performances. 
Thus, we bound the control effort $\|\tilde{d}(t)\| \leq \mu$ along the trajectory for any initial condition $\tilde{x}(0)$ inside the ellipsoid $\tilde{x}(0)^{T} P \tilde{x}(0)$ by means of the following additional LMIs

$$
\left[\begin{array}{cc}
1 & x(0)^{T} \\
x(0) & W
\end{array}\right]<0, \quad\left[\begin{array}{cc}
W & Y^{T} \\
Y & \mu^{2} I
\end{array}\right]<0
$$

where again $W=P^{-1}$ and $Y=F W$.

The previous design LMI are used to obtain robust, efficient control laws in next section where we instantiate the parameters that correspond to the experimental prototype.

\section{CONTROL DESIGN AND SimUlation RESUltS}

In this section, we describe the LMI control procedure applied to both the monovariable model of the cascade boost converter (4) and the multivariable model (7), taking into account their corresponding polytopic uncertainties (10) and (13), respectively.

The procedure consists of finding the feedback gain $F$ such that the current disturbance rejection is maximized, that is minimizing the parameter $\gamma$ in the LMI (29). The minimization is subject to constraints on stability, which corresponds with LMI (24), on pole placement (30)-(32), and on control effort LMIs (33), for each vertex of the polytopic representation of the model. We chose as control parameters those of table I. The converter parameters are shown in table II. Thus, the control design procedure can be expressed by means of the following optimization program

$$
\min _{Y, W} \gamma \text { subject to (28)- (32) } \forall\left\{\varsigma_{i}\right\}, i=1, \ldots, L
$$

The optimization program (34) is convex and can be readily solved by standard interior-point methods using Matlab [12]. Using this procedure ensures that either the optimum is rapidly achieved or the infeasibility is promptly detected.

For the monovariable case, the optimal feedback gain vector corresponds to

$$
F_{\text {mono variable }}=\left[\begin{array}{lllll}
-0.1359 & 0.1760 & -0.0661 & -0.1359 & -64.8178
\end{array}\right]
$$

This controller ensures a $H_{\infty}$ gain of the output voltage with respect to the output current disturbance of $\gamma=3.51(10.9 \mathrm{~dB})$

The optimal feedback gain in the multivariable converter model corresponds to

$F_{\text {multivariable }}=\left[\begin{array}{cccccc}-0.1893 & -0.0540 & -0.0218 & -0.1611 & -12.5137 & -108.4642 \\ 0.0355 & 0.0411 & -0.0714 & 0.0272 & 51.9310 & 8.2799\end{array}\right]$

The $H_{\infty}$ gain of the output voltage with respect to the output current disturbance of this controller is $\gamma=2.31$ $(7.2 \mathrm{~dB})$.
TABLE I

CONTROLLERS PARAMETERS

\begin{tabular}{|l|l|}
\hline$\theta$ & $25^{\circ}$ \\
$\alpha$ & $300 \mathrm{~s}^{-1}$ \\
$\mu$ & 15 \\
$r$ & $\frac{2 \pi f_{s}}{10} \mathrm{rad} / \mathrm{s}$ \\
\hline
\end{tabular}

TABLE II

\section{CASCADE BOOST CONVERTER PARAMETERS}

\begin{tabular}{|l|l|}
\hline $\mathrm{V}_{\mathrm{g}}$ & $10 \mathrm{~V}$ \\
$\mathrm{~V}_{\text {ref }}$ & $40 \mathrm{~V}$ \\
$C_{1}, C_{2}$ & $110 \mu \mathrm{F}$ \\
$L_{1}, L_{2}$ & $150 \mu \mathrm{H}$ \\
$R$ & {$[10,50] \Omega$} \\
$D^{\prime}, D^{\prime}$ & {$[0.4,0.6]$} \\
$f_{s}$ & $120 \mathrm{kHz}$ \\
\hline
\end{tabular}

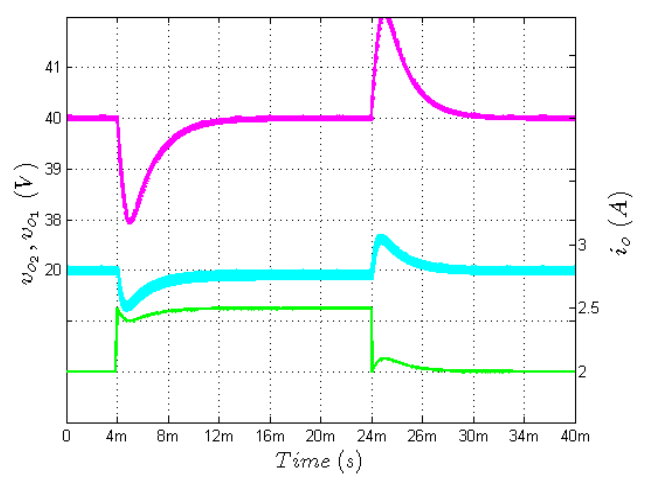

Fig. 2. Simulated response of the monovariable cascade boost converter under a load step transient of $0.5 \mathrm{~A}$.

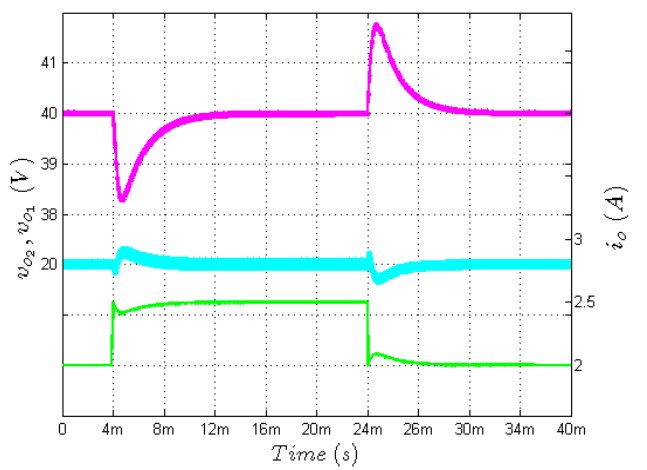

Fig. 3. Simulated response of the multivariable cascade boost converter under a load step transient of $0.5 \mathrm{~A}$.

These values of $H_{\infty}$ gain, show a better performance of the multivariable approach. This behavior is corroborated by PSIM simulations. Also, it can be appreciated that the settling time is smaller than $13.3 \mathrm{~ms}$ as expected since the minimum decay rate is $\alpha=300$. As we have chosen $\theta=25 \sqsubset$ according to table I, the minimum damping ratio expected is $\zeta=0.42$. 


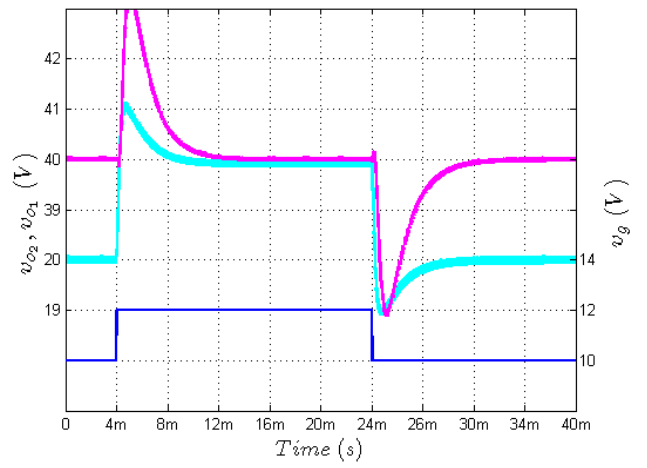

Fig. 4. Simulated response of the monovariable cascade boost converter for step perturbations in the input voltage.

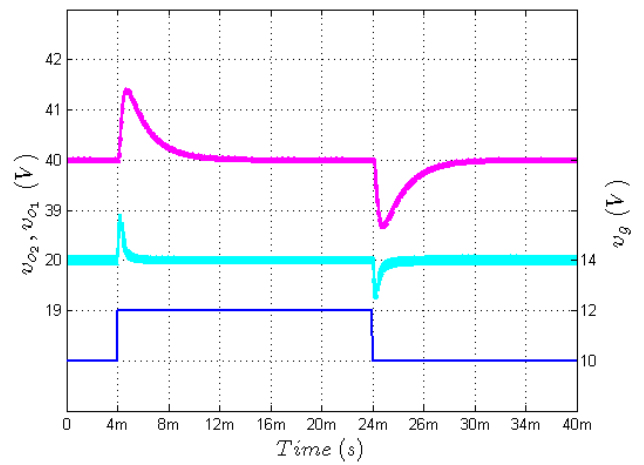

Fig. 5. Simulated response of the multivariable cascade boost converter for step perturbations in the input voltage.

Fig. 2 depicts waveforms of the output current $i_{o}$ and voltages $v_{o 1}$ and $v_{o 2}$ of the monovariable converter controlled by feedback gain (35). The waveforms show an abrupt change from $2 \mathrm{~A}$ to $2.5 \mathrm{~A}$ of the output current $i_{o}$ at $\mathrm{t}$ $=4 \mathrm{~ms}$, and the opposite transition at $\mathrm{t}=24 \mathrm{~ms}$. It can be appreciated that both settling time and damping ratio meet with their design bounds.

The same current disturbance is simulated for the multivariable cascade converter and the waveforms are depicted in Fig. 3. It can be appreciated that the settling time and damping ratio meet the design bounds. Moreover, the disturbance rejection is better in Fig. 3 .

The same current disturbance is simulated for the multivariable cascade converter and the waveforms are depicted in Fig. 3. It can be appreciated that the settling time and damping ratio meet the design bounds. Moreover, the disturbance rejection is better in Fig. 3 .

Figs. 4 and 5 illustrate the responses of voltages $v_{o 1}$ and $v_{o 2}$ to an input voltage variation in the monovariable and multivariable cases, respectively. The input voltage $v_{g}$ changes abruptly from $10 \mathrm{~V}$ to $12 \mathrm{~V}$ at $\mathrm{t}=4 \mathrm{~ms}$ and returns to $10 \mathrm{~V}$ at t $=24 \mathrm{~ms}$. Again, the waveforms comply with the expected performances of settling time and damping ratio. Also, disturbance is better rejected in the multivariable case.

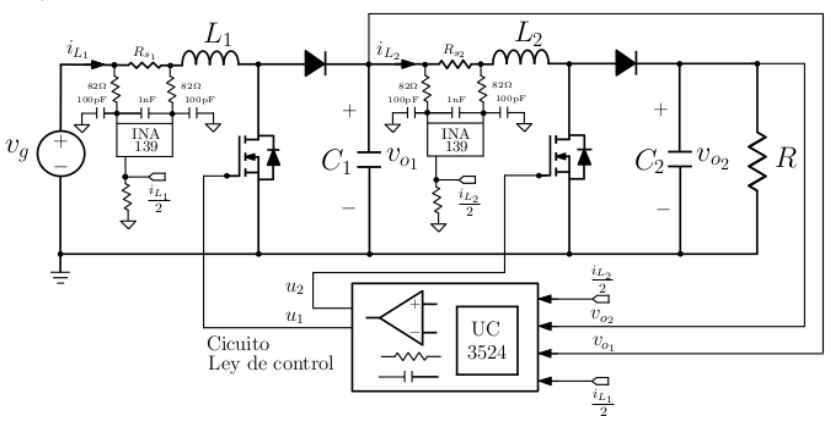

Fig. 6. Circuital diagram of the dc-dc cascade boost converter.

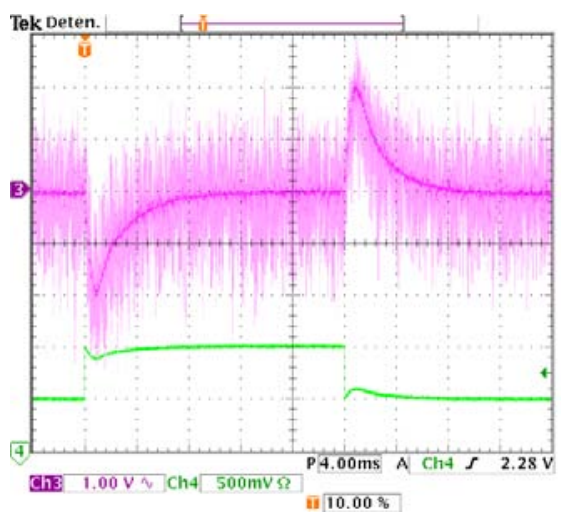

(a) Voltage response $V_{o 2}$.

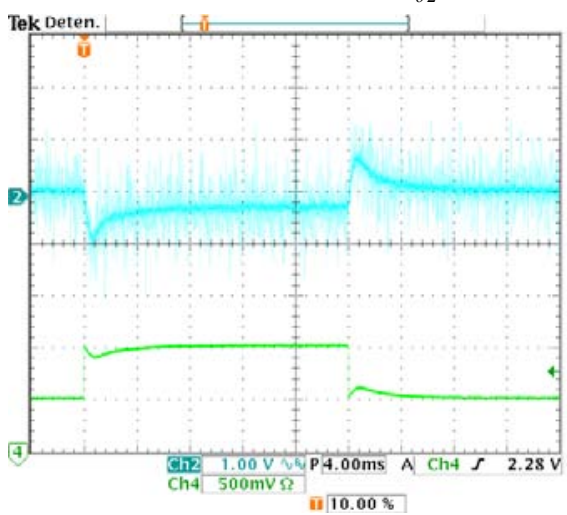

(b) Voltage response $v_{01}$.

Fig. 7. Experimental response of the monovariable.

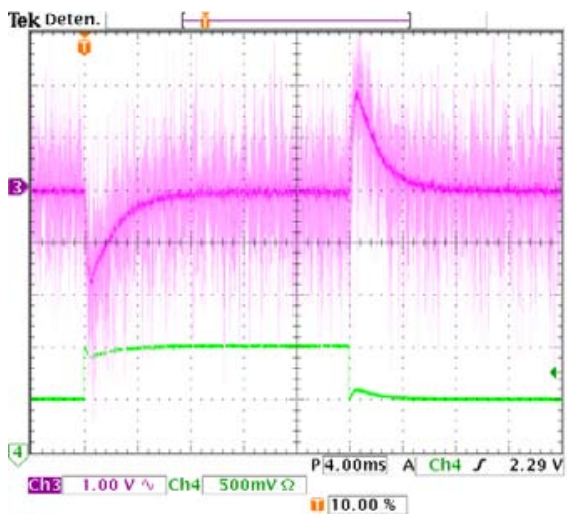

(a) Voltage response $V_{02}$. 


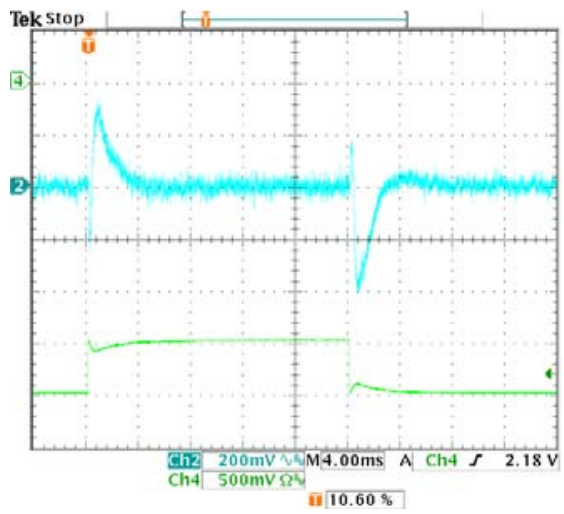

(b) Voltage response $v_{01}$.

Fig.8. Experimental response of the multivariable cascade boost converter to a load step transient of $0.5 \mathrm{~A}$.

\section{EXPERIMENTAL RESULTS}

In this section, we corroborate the previous derivations and simulated waveforms with experimental results. We have implemented a prototype in accordance with tables I and II. The prototype scheme is depicted in Fig.6, where the current is measured by means of shunt resistances $R_{s 1}$ and $R_{s 2}$ of 10 $\mathrm{m} \Omega$ and two current shunt monitors INA 139. The controller details of the monovariable and multivariable controllers, according to (34) and (35), are shown in Figs. 9a and 9b, respectively

Figs. 10 and 11 show the responses to an input voltage change from $10 \mathrm{~V}$ to $12 \mathrm{~V}$. These experimental results and the previous simulated waveforms of figures 4 and 5 are also in very good agreement.

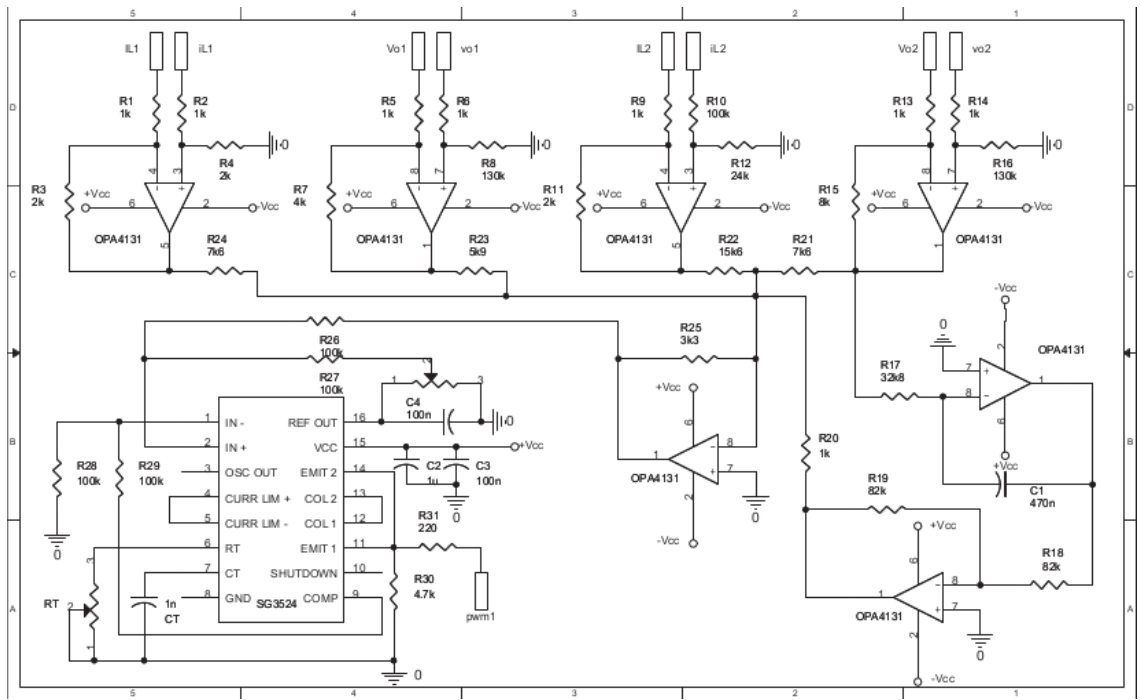

(a) Monovariable case.

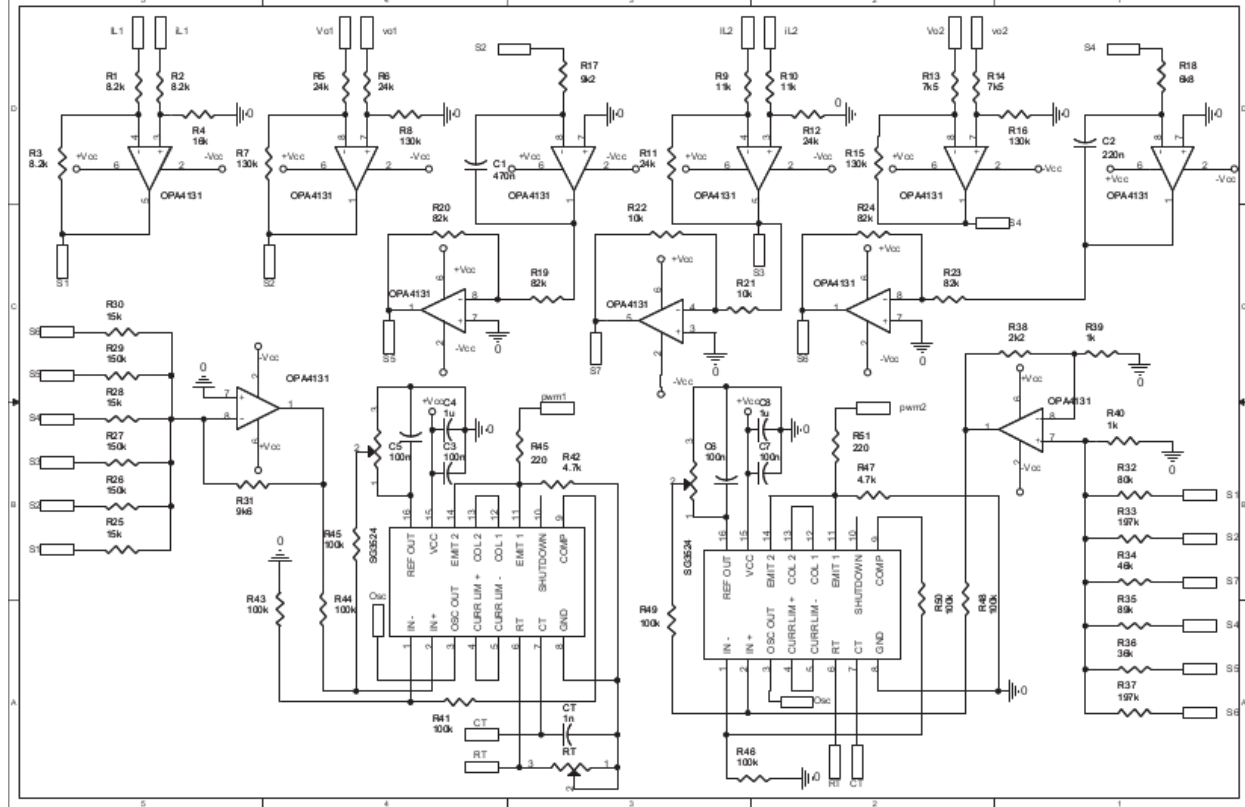

(b) Multivariable case.

Fig. 9. Schematic diagrams of the proposed controllers. 


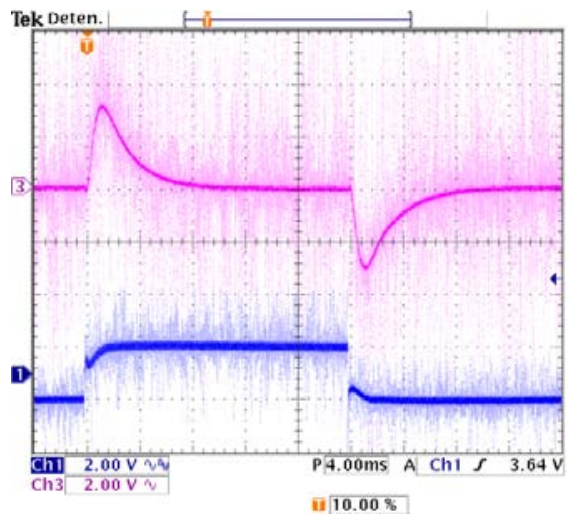

(a) Voltage response $v_{o 2}$.

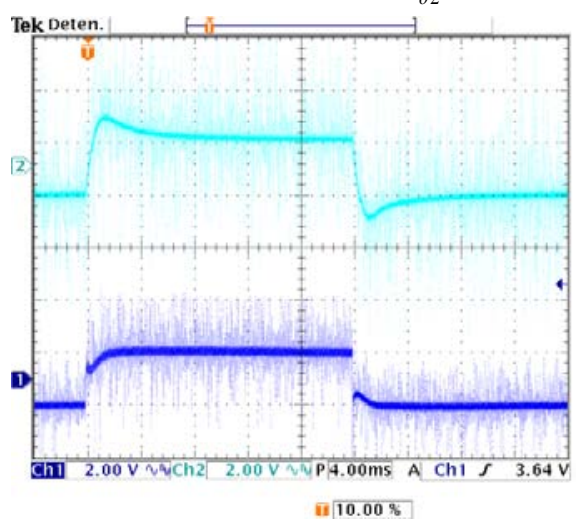

(b) Voltage response $v_{01}$

Fig. 10. Experimental response of the monovariable cascade boost converter to input voltage variations.

\section{CONCLUSIONS}

In the paper, we analyze two averaged models of dc-dc cascade boost converters. The model is monovariable when the switch signal is the same in each stage. On the contrary, the model is multivariable when the switch signal is different for each stage. The models take into account parametric uncertainty by means of a polytopic representation. Then, after reviewing some design constraints in LMI control design, we apply the LMI robust control to the monovariable and multivariable models with the objective of maximizing the output-current disturbance rejection. Finally, we corroborate the procedure by means of experimental measures which show a good agreement with the analytical derivations and the simulated waveforms.

The multivariable model performs better; that is, it has a better disturbance rejection at the expense of a slightly more complex controller. The method can be extended to more stages connected in cascade, thus exploiting all the degrees of freedom of the plant. Also, the proposed method can be readily extended to other converter topologies.

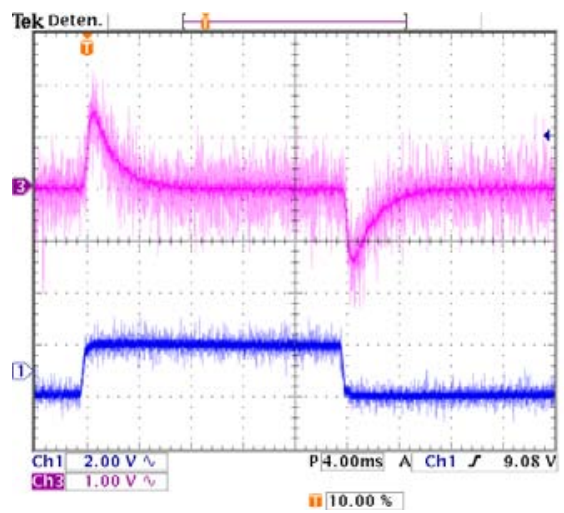

(a) Voltage response $V_{02}$.

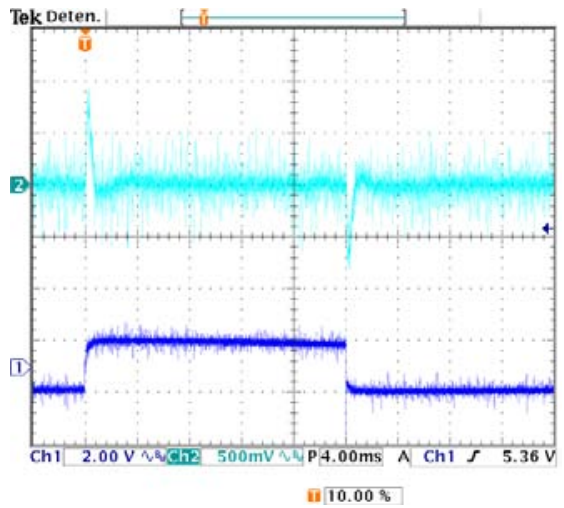

(b) Voltage response $v_{01}$.

Fig. 11. Experimental response of the multivariable cascade boost converter to input voltage variations.

\section{ACKNOWLEDGMENT}

This work was partially supported by the Spanish Ministerio de Educación y Ciencia under grant no. DPI2010-16481

\section{REFERENCES}

[1] D. Maksimovic and S. Cuk, "Switching converters with wide DC conversion range,” IEEE Trans. Power Electron., Vol. 6, No. 1, pp. 151-157, Jan. 1991.

[2] J. A. Morales-Saldaña, J. Leyva-Ramos, E. E. Carbajal-Gutierrez, and M. G. Ortiz-Lopez, “Average current-mode control scheme for a quadratic buck converter with a single switch," IEEE Trans. Power Electron., Vol. 23, No. 1, pp. 485-490, Jan. 2008.

[3] G. R. Walker and P. C. Sernia, "Cascaded DC-DC converter connection of photovoltaic modules," IEEE Trans. Power Electron., Vol. 19, No. 4, pp. 1130-1139, Jul. 2004.

[4] S. Boyd, L. E. Ghaoul, E. Feron, and V. Balakrishnan, "Linear Matrix Inequalities in Systems and Control Theory," Vol. 15 of Studies in Applied and Numerical Mathematics, SIAM, Philadelphia, 1994.

[5] R. Middlebrook and S. Cuk, "A general unified approach to modeling switching-converter power stages," in Rec. IEEE Power Electronics Specialists Conference, pp. 18-34, Cleveland, Jun. 1976.

[6] C. Olalla, R. Leyva, A. El Aroudi, and I. Queinnec, "Robust LQR control for PWM converters: An LMI 
approach,” IEEE Trans. Ind. Electron., Vol. 56, No. 7, pp. 2548-2558, Jul. 2009.

[7] C. Olalla, R. Leyva, A. El Aroudi, P. Garces, and I. Queinnec, "LMI robust control design for boost PWM converters,” IET Power Electronics, Vol. 3, No. 1, pp. 75-85, Jan. 2010.

[8] J. A. Morales-Saldana, R. Galarza-Quirino, J. Leyva-Ramos, E. E. Carbajal-Gutierrez, and M. G. Ortiz-Lopez, "Multiloop controller design for a quadratic boost converter," IET Electric Power Applications, Vol. 1, No. 3, pp. 362-367, May 2007.

[9] R. W. Erickson and D. Maksimovic, Fundamental of Power Electronics, $2^{\text {nd }}$ Ed., Kluwer Academic Publisher, Norwell, Massachusetts, 2001

[10] A. Ríos-Bolívar and G. Garcia, "A robust filters for fault detection and diagnosis: An Ho optimization approach," in Proceeding of European Control Conference, pp. 132-137, Porto, Sep. 2001.

[11] M. Chilali and P. Gahinet, øHdesign with Pole Placement Constraints: An LMI Approach," IEEE Transactions on Autom. Control, Vol. 41, No. 3, pp. 358-367, Mar. 1996.

[12] P. Gahinet, LMI Control Toolbox for Use with Matlab, Natick, MA: The Mathworks, Inc., 1995

[13] J.-P. Lee, B.-D. Min, T.-J. Kim, D.-W. Yoo, and J.-Y. Yoo, "Input-series-output-parallel connected DC/DC converter for a photovoltaic PCS with high efficiency under a wide load range,” Journal of Power Electronics, Vol. 10, No. 1, pp. 9-13, Jan. 2010.

[14] M.-G. Kim and Y.-S. Jung, "A novel soft-switching two-switch flyback converter with a wide operating range and regenerative clamping," Journal of Power Electronics, Vol. 9, No. 5, pp. 772-780, Sep. 2009.

[15] R. D, Middlebrook, “Transformerless DC-to-DC converters with large conversion ratios," IEEE Trans. Power Electron., Vol. 3, No. 4, pp. 484-488, Oct. 1988.

[16] H.-L. Do, "Zero-voltage-switching boost converter using a coupled inductor,” Journal of Power Electronics, Vol. 11, No. 1, pp. 16-20, Jan. 2011.

[17] V. F. Montagner, R. C. L. F. Oliveira, V. J. S. Leite, and P. L. D. Peres, "LMI approach for $\mathrm{H} \infty$ linear parameter-varying state feedback control," IEE Proceedings - Control Theory and Applications, Vol. 152, No. 2, pp. 195- 201, Mar. 2005.

[18] R. Leyva, C. Olalla, I. Queinnec, S. Tarbouriech, C. Alonso, and L. Martinez-Salamero, "Passivity - based control for large - signal stability of high - order switching converters," Asian Journal of Control, Vol. 14, No. 2, pp. 1934-6093, Mar. 2012

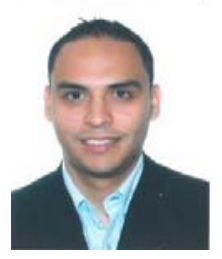

Carlos Andrés Torres-Pinzón received the Ingeniero Electricista and Master en Ingeniería Eléctrica degrees, from the Universidad Tecnológica de Pereira, Pereira, Colombia, in 2006 and in 2008, respectively, and the Master en Ingeniería Electrónica degree from the Universitat Rovira i Virgili de Tarragona, Tarragona, Spain, in 2009. He is currently working toward the Ph.D. degree in the Departament d'Enginyeria Electrònica, Elèctrica i Automàtica, Escola Tècnica Superior d'Enginyeria, Universitat Rovira i Virgili de Tarragona, Tarragona, Spain. His main research interests include robust control and power converters design.

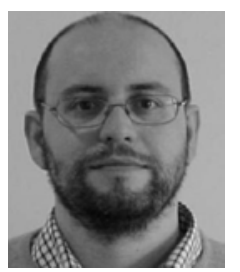

Roberto Giral received the B.S. degree in Ingeniería Técnica de Telecomunicación, the M.S. degree in Ingeniería de elecomunicación, and the Ph.D. (Hons.) degree from the Universitat Politècnica de Catalunya, Barcelona, Spain, in 1991, 1994, and 1999, respectively. He is currently an Associate Professor at the Departament d'Enginyeria Electrònica, Elèctrica i Automàtica, Escola Tècnica Superior d'Enginyeria, Universitat Rovira i Virgili, Tarragona, Spain, where he is working in the field of power electronics.

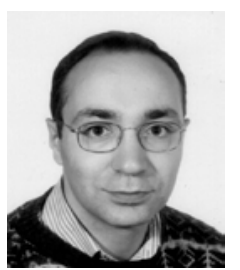

Ramon Leyva received the Telecommunication Engineering and Ph.D. degrees from the Universitat Politècnica de Catalunya, Barcelona, Spain, in 1992 and 2000, respectively. He is currently an Associate Professor with the Departament d'Enginyeria en Electrònica, Elèctrica i Automàtica, Universitat Rovira i Virgili, Tarragona, Spain. From March 2002 to March 2003, he held a Visiting Scholarship with the Laboratoire d'Analyse et d'Architecture des Systèmes, Centre National de la Recherche Scientifique, Université de Toulouse, Toulouse, France. His research task is in the field of nonlinear and robust control of power switching converters. Dr. Leyva serves as Reviewer for several IEEE and Institution of Engineering and Technology scientific publications. 